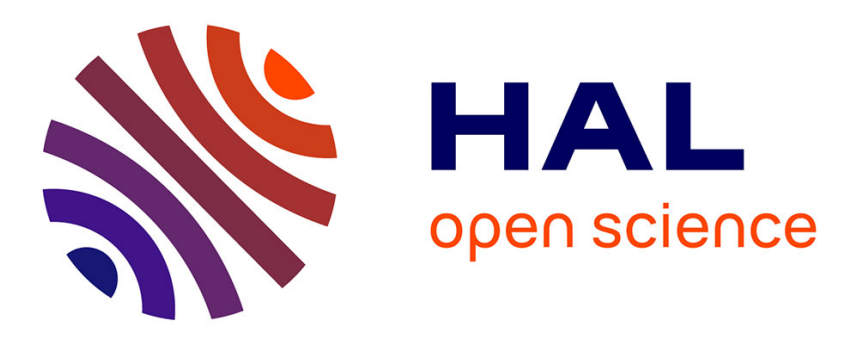

\title{
Backside Laser Testing of Single-Event Effects in GaN-on-Si Power HEMTs
}

\author{
C. Ngom, Vincent Pouget, M. Zerarka, F. Coccetti, Antoine Touboul, M. \\ Matmat, O. Crepel, S. Jonathas, G. Bascoul
}

\section{- To cite this version:}

C. Ngom, Vincent Pouget, M. Zerarka, F. Coccetti, Antoine Touboul, et al.. Backside Laser Testing of Single-Event Effects in GaN-on-Si Power HEMTs. IEEE Transactions on Nuclear Science, 2021, 68 (8), pp.1642-1650. 10.1109/TNS.2021.3081485 . hal-03428144

\section{HAL Id: hal-03428144 \\ https://hal.science/hal-03428144}

Submitted on 15 Nov 2021

HAL is a multi-disciplinary open access archive for the deposit and dissemination of scientific research documents, whether they are published or not. The documents may come from teaching and research institutions in France or abroad, or from public or private research centers.
L'archive ouverte pluridisciplinaire HAL, est destinée au dépôt et à la diffusion de documents scientifiques de niveau recherche, publiés ou non, émanant des établissements d'enseignement et de recherche français ou étrangers, des laboratoires publics ou privés. 


\title{
Backside Laser Testing of Single-Event Effects in GaN-on-Si Power HEMTs
}

\author{
C. Ngom, V. Pouget, M. Zerarka, F. Coccetti, A. Touboul, M. Matmat, O. Crepel, S. Jonathas and G. Bascoul
}

\begin{abstract}
We present backside laser testing of GaN power devices on Si substrate using optical parameters compatible with three-photon absorption in GaN and single-photon absorption in the substrate. The laser/device interaction is described. Two different kinds of transients are observed at the gate electrode and analyzed. The technique allows identifying the sensitive regions of the devices and generating destructive events.
\end{abstract}

Index Terms - Single-event effects, GaN, laser testing, threephoton absorption

\section{INTRODUCTION}

$\mathrm{G}$ ALLIUM nitride $(\mathrm{GaN})$ high electron mobility transistors (HEMTs) have encountered significant interest for power applications in the recent years [1]. They can be used in highvoltage operation thanks to the wide band gap and the high critical electric field [2] of GaN with reduced power losses when compared to $\mathrm{Si}$ devices. Different commercial technologies and references of GaN HEMTs are now available on the market, the most common being Normally-Off HEMTs using GaN-on-Silicon substrate technologies [3].

Single event effects (SEE) induced by ionizing particles are a major reliability concern for any power device in space and aeronautic applications, and the SEE susceptibility of $\mathrm{GaN}$ HEMTs must be evaluated. This is commonly done using accelerated particle beams, such as heavy ions [4]-[6]. Pulsedlaser testing can also be used on $\mathrm{Si}$ technologies to extract the safe operating area (SOA) [7], [8] or the sensitive regions and volumes of power devices using single-photon absorption (SPA) or two-photon absorption (2PA) [9], [10]. 2PA has also been used on $\mathrm{SiC}$ devices [11], [12], another wide bandgap technology. On GaN technologies, previous works have demonstrated the possibility to inject charge on test structures from the front-side using ultraviolet (UV) laser pulses for single-photon absorption [13] and visible light wavelength for two-photon absorption [14], [15]. On commercial parts, contact balls and metal layers will most often prevent using the frontside approach, and UV light would be completely absorbed by the Si substrate if focused through the backside. Other works have also investigated the focused pulsed X-ray technique on $\mathrm{GaN}$ devices [16].

Manuscript received March 1st, 2021.

This work was supported in part by the FELINE PIA project.

C. Ngom is with IRT Saint Exupery, Toulouse, and IES, University of Montpellier, France. V. Pouget is with IES, University of Montpellier, CNRS, France. M. Zerarka, F. Coccetti, M. Matmat are with IRT Saint Exupery, Toulouse, France. A. Touboul is with IES, University of Montpellier, France, O. Crepel is with IRT Saint Exupery and Airbus, Toulouse, France. S. Jonathas is with Pulscan, Gradignan, France. G. Bascoul is with CNES, Toulouse, France.
In this paper, we investigate the backside laser testing approach on commercial references of $\mathrm{GaN}-\mathrm{on}-\mathrm{Si}$ power devices. The possible absorption mechanisms are estimated and the transmission through the backside stack of layers is calculated. Experimental results, including 2D-mappings and depth profiles, using optical parameters compatible with threephoton absorption in the GaN layer are presented and discussed.

\section{DEVICES UNDER TEST}

The devices under test (DUTs) are two commercial references of Normally-Off GaN-on-Si HEMTs. Their structures, extracted from physical analysis, are presented in Fig. 1. A two-dimensional electron gas (2DEG) is created in the $\mathrm{GaN}$ channel layer close to the $\mathrm{AlGaN} / \mathrm{GaN}$ heterojunction. Buffer layers ensure the lattice mismatch transition between the Si substrate and the GaN layer. A p-doped GaN cap on the top of the $\mathrm{AlGaN}$ barrier allows the enhancement mode operation, with a Schottky-contact Gate located between the Drain and Source ohmic contacts.

a)

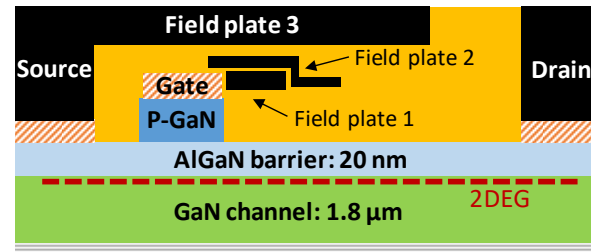

AlGaN/GaN Superlattice: $750 \mathrm{~nm}$

AIGaN : $1.6 \mu \mathrm{m}$

AIN: $207 \mathrm{~nm}$

Si substrate
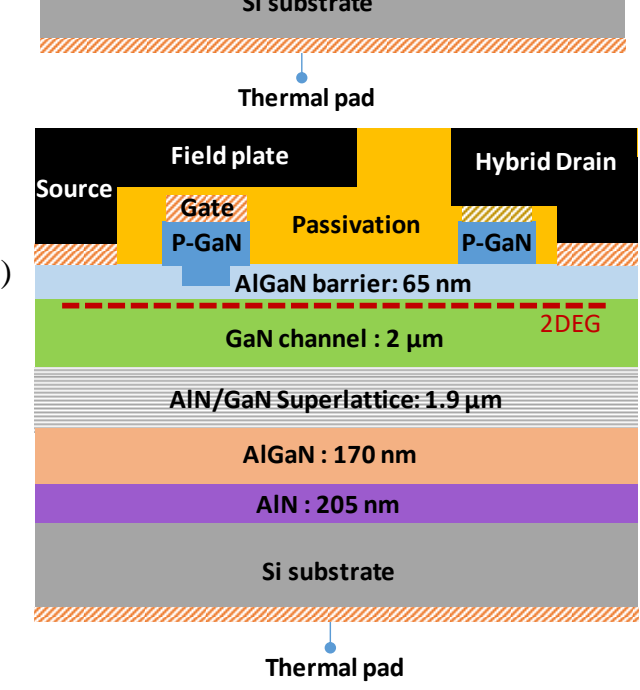

Fig. 1. Device under test layers stack: a) Ref A, b) Ref B. 
In addition to the classical source-connected field plate (FP3 in Fig. 1) [17], reference A presents two field plates FP1 and FP2 close to the gate that could either be floating or connected to the gate [18],[19].

Reference B has a recessed gate, a field plate connected to the source and a hybrid drain formed by an additional gate-like structure electrically connected to the drain [20], [21].

Electrical specifications and layer thicknesses extracted from microsection analysis are presented in Tables I and II, respectively.

TABLE I

DUT ELECTRICAL SPECIFICATIONS

\begin{tabular}{llll}
\hline \hline Parameter & Symbol & Reference A & Reference B \\
\hline $\begin{array}{l}\text { Drain-to-source breakdown } \\
\text { voltage }\end{array}$ & $\mathrm{BV}_{\mathrm{DS}}$ & $650 \mathrm{~V}$ & $600 \mathrm{~V}$ \\
$\begin{array}{l}\text { Continuous drain current } \\
\text { Gate threshold voltage }\end{array}$ & $I_{D S(M A X)}$ & $30 \mathrm{~A}$ & $26 \mathrm{~A}$ \\
$\begin{array}{l}\text { Drain-to-source ON } \\
\text { resistance }\end{array}$ & $V_{G S_{-} T H}$ & $1.7 \mathrm{~V}$ & $1.2 \mathrm{~V}$ \\
$\begin{array}{l}\text { Drain leakage current } \\
\text { Reverse gate leakage current }\end{array}$ & $50 \mathrm{~m} \Omega$ & $56 \mathrm{~m} \Omega$ \\
\hline \hline
\end{tabular}

TABLE II

DUT LAYERS STACK

\begin{tabular}{lll}
\hline \hline & Reference A & Reference B \\
\hline \# tested samples & 4 & 3 \\
AlGaN barrier & $20 \mathrm{~nm}$ & $65 \mathrm{~nm}$ \\
GaN channel & $1.78 \mu \mathrm{m}$ & $2 \mu \mathrm{m}$ \\
Buffer 1 & $5 \mathrm{~nm} \_A l G a N / 20 \mathrm{~nm} \_G a N$ & $5 \mathrm{~nm} \_A l N / 20 \mathrm{~nm} \_G a N$ \\
& $(x 30)$ & $(x 80)$ \\
Buffer 2 & AlGaN $1.56 \mu \mathrm{m}$ & AlGaN 170 nm \\
Buffer 3 & AlN 207 nm & AlN 205 nm \\
Si substrate & \multicolumn{2}{c}{$150 \mu \mathrm{m}$ (after sample preparation) } \\
\hline \hline
\end{tabular}

The $\mathrm{Si}$ substrates have an initial thickness of several hundreds of micrometers, and a metal layer is present on the backside for electrical grounding and thermal sinking. For laser testing, the substrates were locally thinned down to $150 \mu \mathrm{m}$ using micromachining. The backside surface was polished to standard optical mirror quality as required for backside laser testing [22]. The exact residual substrate thickness of each sample was measured optically. More than half of the backside metal area in place was preserved to reduce the perturbation of the substrate electrical and thermal contact. Removing the full Si substrate thickness was not considered as this could lead to significant perturbation of the electrostatic equilibrium state of the device due to the piezoelectric nature of the materials. Devices were carefully characterized before and after sample preparation to observe any degradation of the static electrical characteristics due to mechanical constraints or relaxation of the substrate during preparation. In particular, IV curves at low $\mathrm{V}_{\mathrm{DS}}$ were extracted to observe any leakage current degradation. Only the devices that presented negligible or no degradation of their characteristics were kept for the laser testing campaign presented in this work.

\section{OPTICAL MODELLING}

Laser testing for single-event effects requires to optically inject electron-hole pairs in the active layer of the device. This requires, first, to identify a possible absorption mechanism at a given wavelength and, second, to estimate the possibility for photons at this wavelength to reach the active layer. As a support for this section, Table III presents the bandgap energy of the different materials of interest.

TABLE III

LAYER BANDGAP ENERGIES

\begin{tabular}{ll}
\hline \hline Material & Bandgap energy $(\mathrm{eV})$ \\
\hline $\mathrm{GaN}$ & 3.42 \\
$\mathrm{Al}_{0.28} \mathrm{Ga}_{0.72} \mathrm{~N}$ & 4.2 \\
$\mathrm{AlN}$ & 6.2 \\
Silicon & 1.12 \\
\hline \hline
\end{tabular}

\section{A. Absorption mechanisms}

We consider a laser beam impinging through the backside and focused in the GaN channel. Considering that the photons have to go through the thick Si substrate before reaching the GaN layer, SPA is not an option. Indeed, photons with the energy required to create pairs in $\mathrm{GaN}(3.42 \mathrm{eV})$ would be completely absorbed in $\mathrm{Si}$ over a short distance $(<1 \mu \mathrm{m})$ [23][24]. Thus, we consider various multi-photon absorption mechanisms noted XPA in the following, where $x$ is the number of photons required to create a single electron-hole pair. Each mechanism has a condition on the photon wavelength given by:

$$
\lambda<\lambda_{x P A-\max }=x \lambda_{g}
$$

where $\lambda \mathrm{g}$ is the wavelength corresponding to the bandgap of $\mathrm{GaN}(362 \mathrm{~nm})$. When this wavelength condition is satisfied, the xPA absorption coefficients of $\mathrm{GaN}$ is given by [25]:

$$
\beta_{x P A}(\hbar \omega)=C_{x} F_{x}\left(\frac{x \hbar \omega}{E_{g}}\right) \frac{\hbar^{x-1} P^{2 x-3}}{n^{x} E_{g}^{4 x-5}}
$$

where $n$ is the refractive index, $E_{g}$ the bandgap energy and $P$ the Kane parameter defined as $P=\hbar \sqrt{E_{p} / 2 m_{0}}$ with $E_{p}$ the Kane energy and $m_{0}$ the electron mass. $C_{x}$ is a dimensionless factor, and $F_{x}$ a dimensionless function that gives the dependency of the absorption coefficient with respect to the wavelength.

Other multiplication factors not presented in (2) should be added to obtain an exact amplitude of the absorption coefficient. They tend to increase the amplitude of the absorption coefficient $\beta_{x P A}$ [25], so (2) provides a lower limit estimation.

Using common assumptions on electron orbital shapes, we estimated $\beta_{X P A}$ for $x=2,3,4$ and 5 at wavelengths slightly below $\lambda_{x P A-\max }$. For each of those absorption mechanisms, we then calculated the laser pulse energy $E_{L}$ required in the active layer for the generated charge distribution in $\mathrm{GaN}$ to have the same amplitude as the one induced in Si by a pulse of $100 \mathrm{pJ}$ at $1064 \mathrm{~nm}$, which are usual values for SPA laser testing of $\mathrm{Si}$ technologies. Note that, due to the underestimation of $\beta_{x P A}$ 
mentioned above, the calculated required energies for xPA mechanisms are worst case values in the sense that they are overestimated. Considering the relationship between the laser energy and the induced peak generation rate [26], the laser energy for each mechanism is obtained using the following equation:

$$
E_{L}=\frac{\pi^{3 / 2} \omega_{0}{ }^{2} \tau}{2}\left(\frac{x E_{\gamma} G_{0}}{\beta_{x P A}}\right)^{1 / x}
$$

where $E_{\gamma}$ is the photon energy, $G_{0_{S P A}}$ is the reference pair generation rate amplitude in silicon, $\omega_{0}$ is the waist of the Gaussian beam and $\tau$ is the pulse duration. The units of $\beta_{x P A}$ are $m^{2 x-3} / W^{x-1}$.

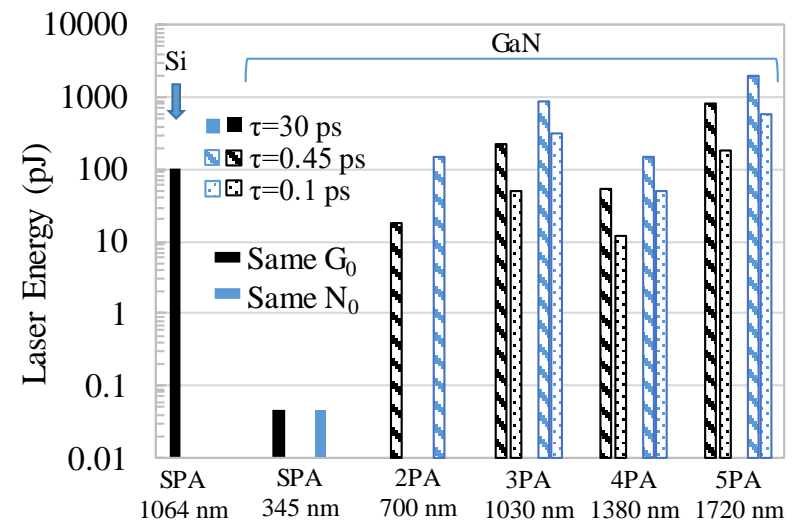

Absorption mechanisms

Fig. 2. Required pulse energy at a given wavelength for each absorption mechanism in $\mathrm{GaN}$ material compared to $\mathrm{SPA}$ in $\mathrm{Si}$, for different laser pulse durations $(\tau)$

Note that $E_{L}$ is the laser pulse energy in the active layer, so its calculation does not include the transmittance of the backside stack (calculated in the next section). The results in Fig. 2 present the energy in the active layer required to obtain either the same generation rate amplitude $\left(G_{0}\right)$ or the same charge distribution amplitude $\left(N_{0}\right)$ as in the silicon reference case. Note that, in the case of a Gaussian time profile for the laser pulse, both amplitudes are related by $N_{0}=\sqrt{\pi} \tau G_{0}$.

Results presented in Fig. 2 show that three-photon absorption (3PA) at $1030 \mathrm{~nm}$ is possible with reasonable pulse energies in the active layer (same order of magnitude as for $\mathrm{Si}$ ) using femtosecond pulse durations, making this mechanism a good candidate for backside laser testing of GaN-on-Si devices. Indeed, this wavelength is commonly available and has a penetration depth in $\mathrm{Si}$ of several hundreds of micrometers, meaning that a significant fraction of photons can reach the $\mathrm{GaN}$ layer through a thinned substrate, whereas in the case of 2PA, photons at $700 \mathrm{~nm}$ would be absorbed in Si over a few micrometers. Note that using a wavelength of $1030 \mathrm{~nm}$ for generating carriers through 3PA will also lead to SPA generation in the $\mathrm{Si}$ substrate. On the one hand, this is interesting in terms of fidelity to reproduce a heavy ion track in the whole stack. However, on the other hand, this obviously makes a complete modeling of the laser-induced charge track more complex. Such modeling is out of the scope of this paper but would be required for accurate estimation of the total generated charge.

\section{B. Transmittance through the backside layers stack}

Results of Fig. 2 are first-order approximations that do not include yet attenuation and multiple reflections in the buffer layers and in the Si substrate. Those effects were modeled using a classical matrix approach [27] in the Gaussian beam approximation. Since the beam is strongly convergent in the substrate (i.e., its Rayleigh length is much shorter than the substrate thickness), the interferences of the multiple reflections occurring in the Si layer will have a negligible effect on the transmittance. Thus, multiple interferences only have to be considered in the much thinner buffer layers.

The calculated stack (buffer and substrate layers) transmittance of the two DUTs is presented in Fig. 3, as well as the transmittance of the $150 \mu \mathrm{m}$ silicon substrate only. Both references exhibit very similar transmittance curves, with only minor differences above $1030 \mathrm{~nm}$. This result indicates, as could be expected, that the transmittance curves are dominated by attenuation in the Si substrate (same thickness for both references), with little contribution of the different thin buffer layers. At $1030 \mathrm{~nm}$, the calculated transmittance is $40 \%$ for both references, with only $3 \%$ of the losses due to the buffer layers. This transmittance of $40 \%$ seems sufficient to produce significant 3PA absorption in the GaN active layer. Note that the calculated transmittance only includes linear mechanisms, and as such, does not include attenuation due to 3PA in the supperlattice layers.

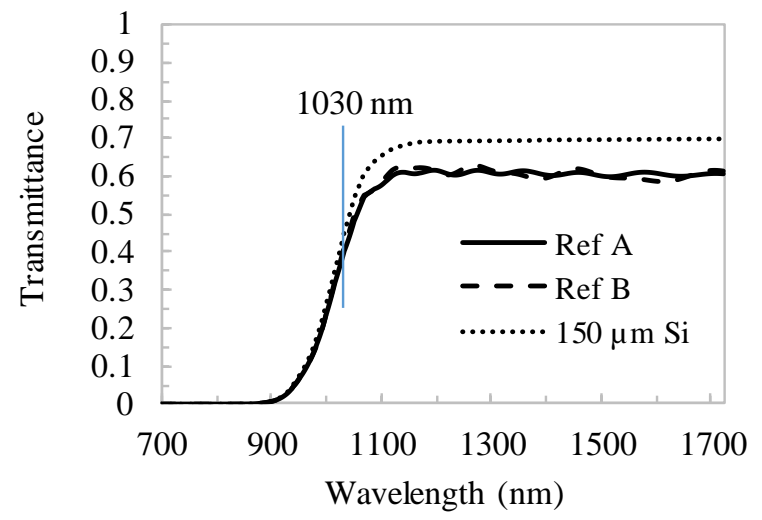

Fig. 3. Backside layer stack transmittance as a function of wavelength.

\section{EXPERIMENTAL SETUP}

Experiments were performed at the laser facility of IES, University of Montpellier. The electrical circuit of the test bench and the experimental setup are shown in Fig. 4.

The laser source wavelength is $1030 \mathrm{~nm}$. The laser pulse duration is $320 \mathrm{fs}$ at the laser output and pulse energies incident on the DUT are lower than $15 \mathrm{~nJ}$. The beam is focused using a $100 \mathrm{X}$ objective lens down to a knife-edge measured $1 / \mathrm{e}^{2}$ spot diameter of $1.0 \mu \mathrm{m}$.

A $100 \mathrm{k} \Omega$ drain resistance and $1 \mathrm{k} \Omega$ gate resistance are used to limit the currents and to allow the observation of voltage transients. The source and the substrate contacts are connected to the ground. 
Static drain and gate currents are continuously monitored during the experiments. Drain and gate voltage transients are captured with a $4 \mathrm{GHz}$ - $20 \mathrm{GS} / \mathrm{s}$ oscilloscope triggered by the laser pulses. To preserve the oscilloscope inputs from destruction by high voltages, all inputs are set in AC high impedance $(1 \mathrm{M} \Omega)$ mode.

Computer-controlled scans are performed while increasing $\mathrm{V}_{\mathrm{DS}}$ from 0 to $\mathrm{V}_{\mathrm{DS}-\mathrm{max}}$, reducing $\mathrm{V}_{\mathrm{GS}}$ from 0 to $-2 \mathrm{~V}$ and increasing the laser pulse energy. The scanned area is frequently shifted to prevent the accumulation of invisible degradation on a same position. For the same reason, the laser pulse triggering is single-shot and, considering the scanning speed, never exceeds $5 \mathrm{~Hz}$.

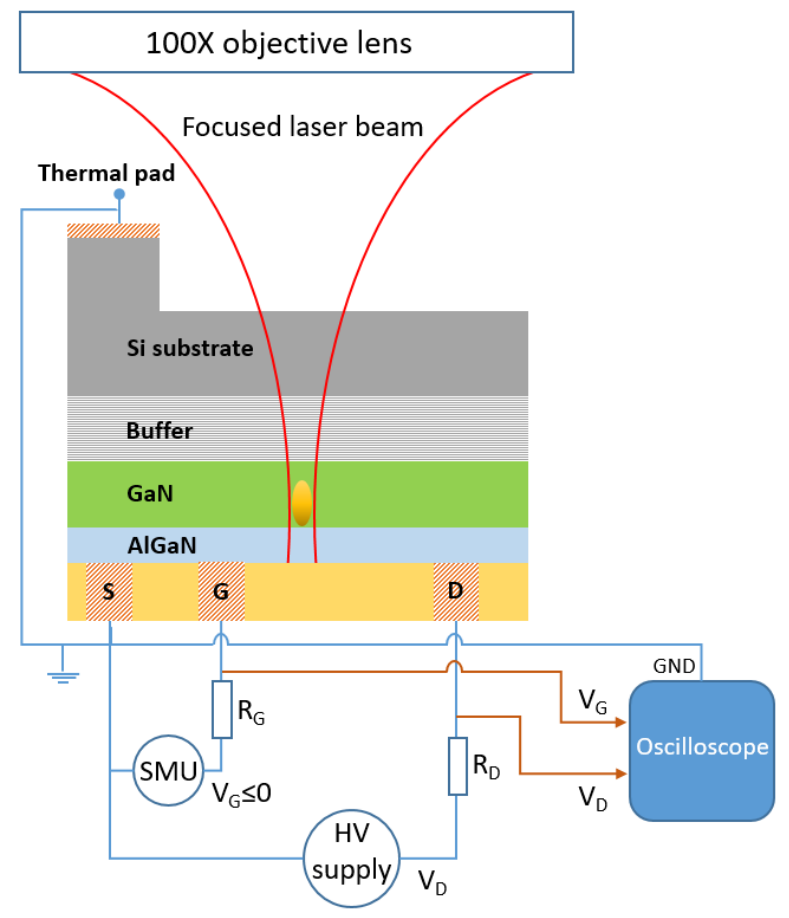

Fig. 4. Experimental setup.

\section{RESULTS AND DISCUSSION}

For both tested references, two categories of transients were observed on the $\mathrm{V}_{\mathrm{GS}}$ oscilloscope channel and are presented in Fig. 5.

Short transients (Fig. 5a.) of a few nanoseconds were observed for any $\mathrm{V}_{\mathrm{DS}}$ above $10 \mathrm{~V}$ and $50 \mathrm{~V}$ respectively for ref $\mathrm{A}$ and B. For $\mathrm{V}_{\mathrm{DS}}$ above $450 \mathrm{~V}$, long transients (Fig. 5b) superimposed with the short ones, with a higher amplitude for ref. A. Both types of transients have small amplitudes in the few $\mathrm{mV}$ to tens of $\mathrm{mV}$ range. Due to bandwidth limitations in the electrical measurement setup, it is not clear if the bipolar shape of the short transients is meaningful or due to distortion by the high impedance of the acquisition channel. Long transients have a more conventional charge collection shape with durations typically around $30 \mu \mathrm{s}$. As will be detailed in the next sections, the short transient amplitudes exhibited a strong dependence with the focus position around the optimal imaging focus whereas the long transients show little variation when focusing in the substrate.
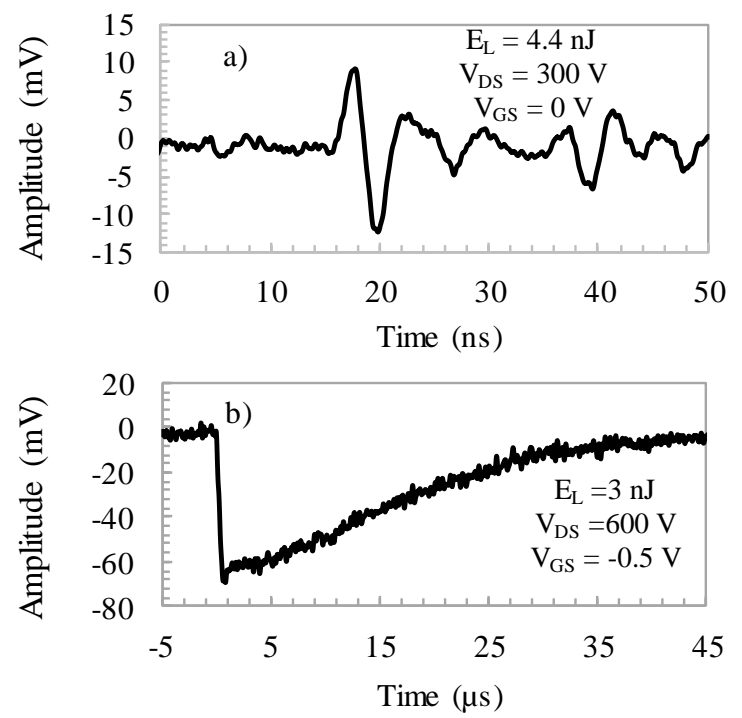

Fig. 5. a) Short time scale transient, b) Long time scale transient, ref. A.

Transients with an amplitude of a few $\mathrm{mV}$ were also observed on the $V_{D S}$ probe but excessive noise of the highvoltage power supply prevented any exploitation of those, so the following results are only based on signals measured at the gate.

\section{A. Short $V_{G S}$ transients}

\section{1) 2D Mapping}

Fig. 6 presents a 2D mapping of the short transients peak-topeak amplitude across one of the many "fingers" of the device for ref. $A$ at $V_{D S}=300 \mathrm{~V}$ and ref. B at $600 \mathrm{~V}$. One can clearly observe that, for reference $\mathrm{A}$, those transients are very localized along the gate contacts on both sides of the source contact. Although the short transients have a very small amplitude, they are sufficient to observe differences between both references. For reference B, besides the gate response, one can also observe an increase of the signal on both sides of the drain contact where the additional gate-like structures are located. At the positions where the signal is minimum, the transient amplitude is nonnegligible.

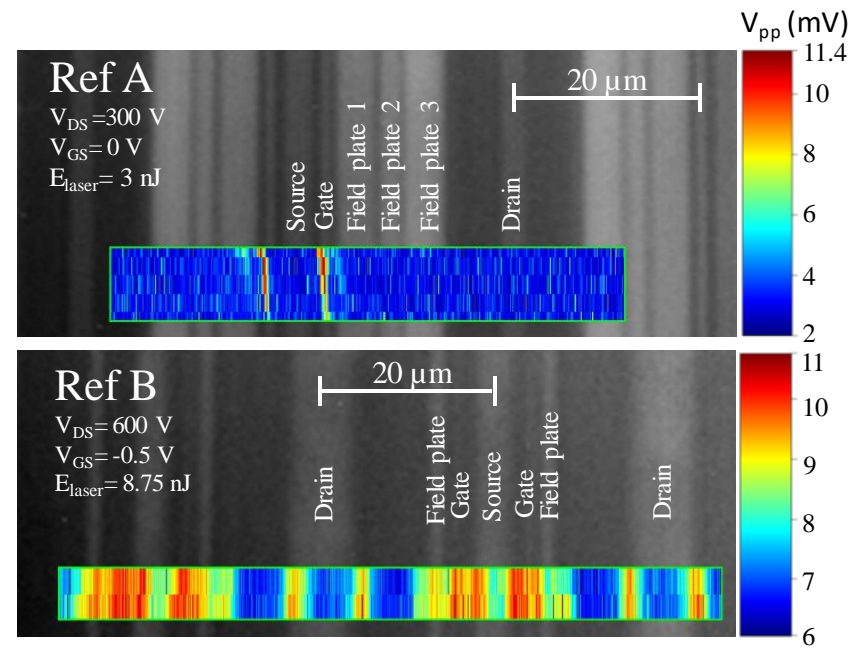

Fig. 6. Map of short transients peak-to-peak amplitude in $\mathrm{mV}$ at laser energy of $3 \mathrm{~nJ}$ on reference A (top) and $8.75 \mathrm{~nJ}$ on reference B (bottom). 
Note that the two mappings presented in Fig. 6 were obtained with different values of the drain voltage and laser energy adjusted to produce very similar amplitudes of the transients. With these particular conditions, the responsive area exhibited by ref. $\mathrm{B}$ is significantly larger. A mapping of ref $\mathrm{A}$ at $\mathrm{V}_{\mathrm{DS}}=$ $600 \mathrm{~V}$ and $\mathrm{V}_{\mathrm{GS}}=-0.5 \mathrm{~V}$, not included because of space considerations, shows that transients appear at any position on the device but the amplitude is still maximum at the gate contacts.

In order to identify the charge generation mechanism at play, we measured the influence of the laser pulse energy and the focus depth on the transient amplitudes.

\section{2) Influence of laser pulse energy}

Fig. 7 presents the short transient amplitude measured with the laser spot positioned at the gate (where the signal is maximum) as a function of the cube of the laser pulse energy. Considering only the points that are above the noise floor at 2 $\mathrm{mV}$, the trend seems roughly linear from $10 \mathrm{~nJ}^{3}$ to $75 \mathrm{~nJ}^{3}$, before a saturation phenomenon appears at higher energies.

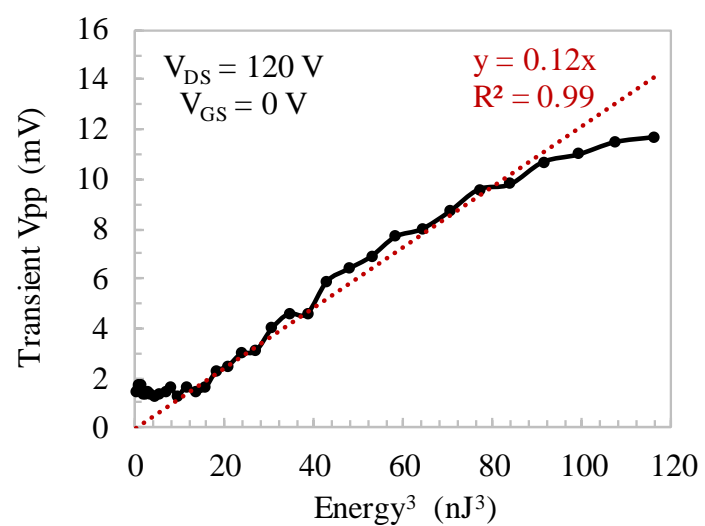

Fig. 7. Short transient peak-to-peak amplitude as a function of pulse energy cube, ref A.

Assuming that the transient peak-to-peak amplitude $V_{p p}$ is proportional to the collected charge $Q_{c}$, the linear section of the curve would be the expected trend for the 3PA mechanism, while the saturation section could be explained by nonlinearities in the pulse propagation through the substrate occurring at higher energies. The $Q_{c}$ to $V_{p p}$ linear relationship could also saturate due to the parasitic elements of the electrical measurement setup. Unfortunately, it was not possible to measure the collected charge to observe this with enough precision due to the bandwidth and noise limitations of our setup.

Now considering that the short transients are induced by 3PA in the GaN layer,it is interesting to note that reflections on metal layers are not expected to play a significant role since 3PA is extremely sensitive to the quality of the optical wave, which is typically distorted by reflections on non-perfectly planar metal contacts. This probably excludes any contribution of reflections in the data of Fig 6.

\section{3) Influence of focus depth}

Fig. 8 shows the strong dependence of the short transient amplitude measured at the gate contact with the focus depth,

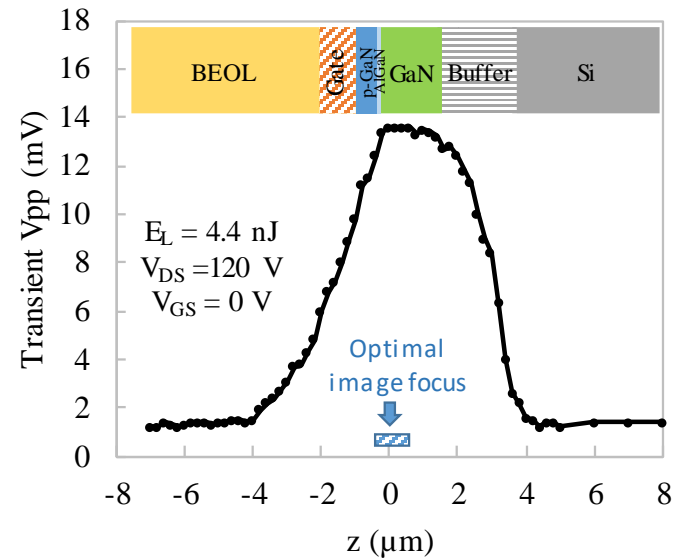

Fig. 8. Transient peak-to-peak amplitude as a function of focus position along Z-axis, ref A.

measured by moving the laser focal spot along the optical axis $Z$. Positive values of $Z$ correspond to a greater distance between the DUT and the objective lens, i.e., the focus spot is moved from the active layer towards the substrate, whereas negative values correspond to a focus position moved towards the dielectric and metal interconnection layers. The origin of the $\mathrm{Z}$ axis is defined as the optimal focus position for imaging the transistor structures. Note that there exists a small uncertainty on the position of this optimal image position due to the $1 \mu \mathrm{m}$ depth-of-field of the objective lens.

The transient amplitude is maximum at the optimal image position, i.e., when focusing the laser spot into the active $\mathrm{GaN}$ layer. The amplitude quickly decreases when the focus point moves away from the active layer. The same trends were observed on both references. This result is compatible with 3PA charge generation in the active layer. Indeed, 3PA generation is expected to be significant only near the focal point of the beam and to decrease rapidly away from this region due to the beam size increase. This phenomenon, which is well-known for 2PA [28], should be even faster for 3PA since the generation rate is then proportional to $w(z)^{-6}$, where $w(z)$ is the beam width profile along the optical axis.

\section{4) Influence of $V_{G S}$ and $V_{D S}$}

Fig. 9 presents the short transient amplitudes as a function of the drain voltage for ref A. One can clearly observe the linear increase of the amplitude with $V_{D S}$, which can be related to the effect of the increasing electrical field on the separation of the

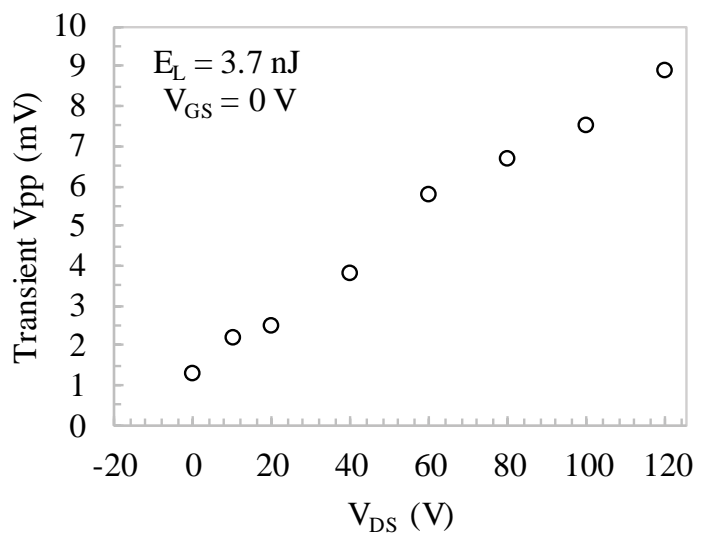

Fig. 9. Short transient peak-to-peak amplitude as a function of $\mathrm{V}_{\mathrm{DS}}$, ref $\mathrm{A}$. 


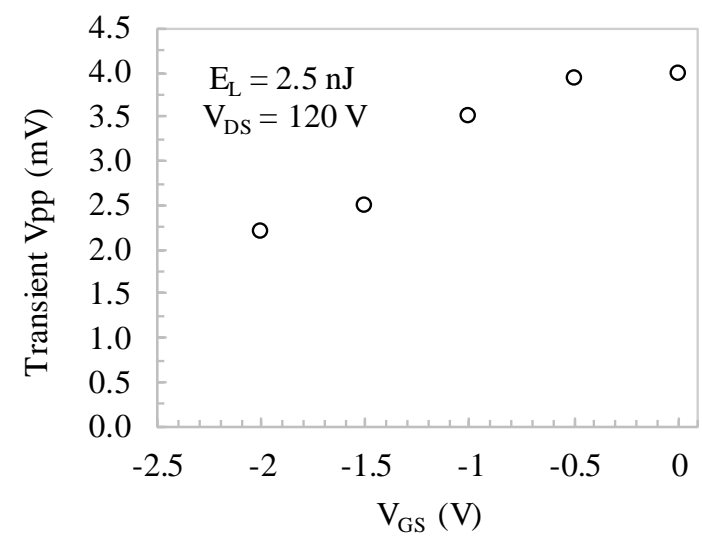

Fig. 10. Short transient peak-to-peak amplitude as a function of $\mathrm{V}_{\mathrm{GS}}$, ref $\mathrm{A}$.

laser generated electron-hole pairs. Similarly, for ref B, the short transient amplitudes was observed to increase with $\mathrm{V}_{\mathrm{DS}}$.

Fig. 10 shows that the transient amplitude also depends on $\mathrm{V}_{\mathrm{GS}}$. At $\mathrm{V}_{\mathrm{GS}}=0 \mathrm{~V}$, the amplitude is maximum and decreases for negative values of $\mathrm{V}_{\mathrm{GS}}$. This behavior could be explained by the fact that the over-blocking condition tends to extend the depopulation of the 2DEG region under the gate, thus increasing the resistive paths for charge collection at the gate.

\section{B. Long $V_{G S}$ transients}

\section{1) 2 D Mapping}

Long transients observed at higher drain voltage exhibited different behaviors as a function of the position on both references.

Fig. 11 (top) presents a 2D-mapping of the long transients collected charge observed at $600 \mathrm{~V}$ on ref A. This mapping presents a significant contrast between the different regions of the device. The maximum absolute signal, in blue color because the transient is negative, is clearly observed in the gate and field-plate 2 and 3 regions between the gate and drain contacts. This can probably be explained by the presence of a highelectrical field in this region that is particularly efficient in splitting electron-hole pairs, as was observed in previous works using focused X-rays [29]. It is interesting to note that the collected charge is lower in the field-plate 1 region, which
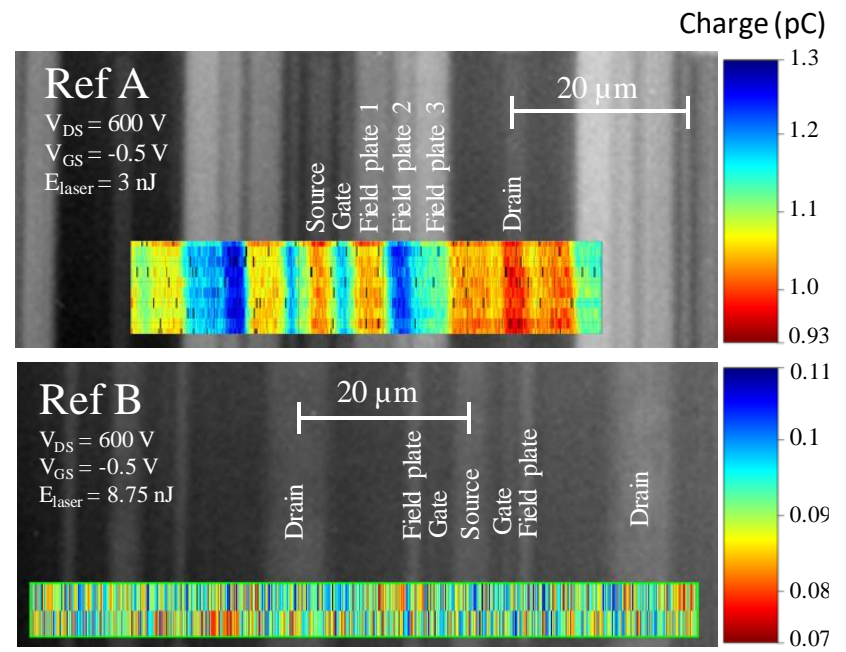

Fig. 11. Mapping of long transients collected charge in $\mathrm{pC}$ at laser energy of $3 \mathrm{~nJ}$ on ref. A (top) and $8.75 \mathrm{~nJ}$ on ref. B (bottom). demonstrates the efficiency of this field plate in locally reducing the electrical field. The difference in collected charge between the field-plate 1 and 2 regions also clearly suggests that those field-plates have different electrical connections: field plate 2 seems to be connected to the gate, while field-plate 1 is most probably floating. It is an interesting feature of the laser technique to be able to provide such information since the structural analysis of the device was not conclusive regarding those field-plates connections.

Surprisingly, a similar 2D-mapping of long transients on reference $\mathrm{B}$ did not present any reproducible contrast as a function of the position (see Fig 11, bottom), with the transient amplitude varying randomly with the laser impact position around values one order of magnitude lower than for ref. A. This spatially random response is unusual in the laser mapping approach and questions the charge generation and collection mechanisms at play for the long transients observed on this device. The beginning of an explanation can be envisioned thanks to the following results.

\section{2) Influence of laser pulse energy}

Fig. 12 presents the collected charge associated with long transients as a function of laser energy. The dotted lines represent linear trend of the solid lines. For ref. A, the collected charge increases linearly from 3 to $6.9 \mathrm{~nJ}$. A similar trend was observed on ref. B, which exhibits lower collected charge. This linear trend seems to indicate that the dominant charge

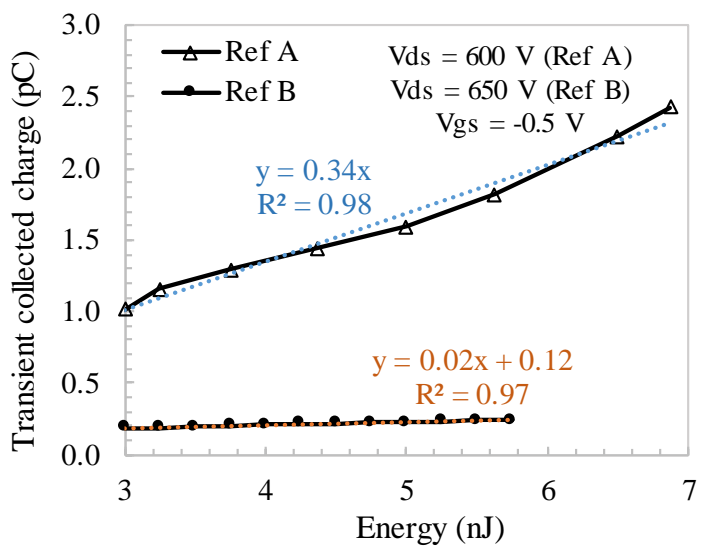

Fig. 12. Long transient collected charge as a function of pulse energy.

generation mechanism for long transients is related to SPA rather than 3PA. This means that the short and long transients result from significantly different charge generation and collection processes, as could be expected from their very different time scales. The small offset in the linear fitting equation of Fig. 12 is not significant and could be related to measurement noise.

\section{3) Influence of focus depth}

Fig. 13 presents the variation of the collected charge associated with long transients with the focus depth. Note the different scale of the horizontal axis with respect to Fig. 8. The collected charge of long transients varies much more slowly with $z$ than the short transient amplitudes. The maximum is obtained when focusing inside the substrate, close to the buffer 


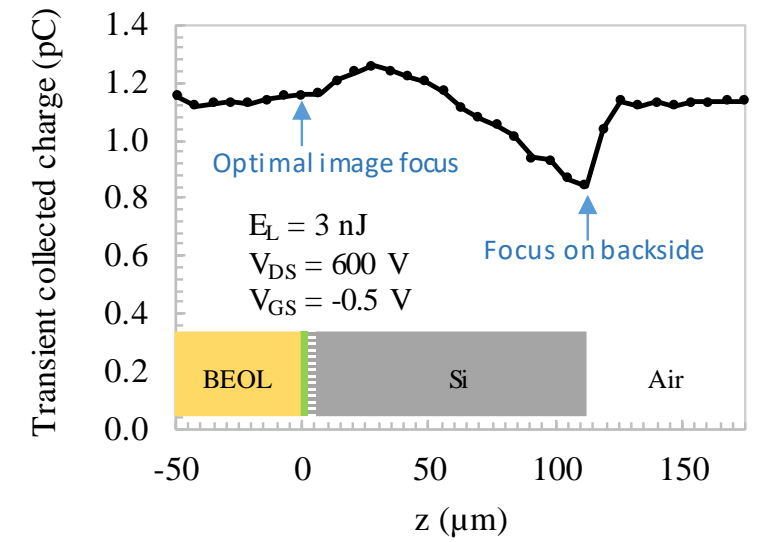

Fig. 13. Long transient collected charge as a function of focus position along $\mathrm{Z}$-axis, ref $\mathrm{A}$.

layers. The slow variation with $z$ and the maximum position confirm that the long transients primarily originate from SPA charge generation in the $\mathrm{Si}$ substrate, rather than 3PA in the active layer. Since charge collection is observed at the gate contact, this implies the existence of a charge transfer mechanism from the substrate to the active layer, through the buffer layers.

For the wavelength used in this work, SPA leads to a penetration depth in Si close to $1 \mathrm{~mm}$, so the charge generation occurs all along the optical axis (i.e., also beyond the focal point) and its profile along the optical path is governed by the beam-waist variation along the path rather than by attenuation. From the maximum position in Fig. 13, the collected charge decreases slowly when moving the focus position towards the backside. This might be explained by the fact that the charge generated close to the buffer layer, where the charge transfer occurs, is spread over a wider area as the beam is focused away from this interface.

A minimum is observed when focusing on the backside surface, which could be related to changes in the backside reflection depending on the focus position.

Considering the different mechanisms at play for short and long transients, the contrast difference for long transients between both references observed in Fig. 11 could then be explained by a less efficient or a more delocalized transfer of charge from the substrate to the channel in ref. B due to its thicker superlattice buffer layer, as seen in Fig. 1. The charge transfer mechanism from the substrate to the GaN layer is still unclear, as it could involve carrier tunneling and/or charge mirroring through the semi-insulating AlN buffer layer. Correlation of the gate transients with transients observed on the drain electrode would probably help in the analysis. However, a full understanding of this mechanism would require a complete TCAD modelling of the layer stacks and is beyond the scope of this paper. This is certainly an interesting mechanism to investigate as it could have serious implications on the SEE sensitivity of those technologies and on the estimation of the sensitive depth for SEE rate calculations.

Another way to contribute to the explanation of those results would be to isolate the effects of 3PA in the active layer from the effect of SPA in the substrate by using different optical parameters for the laser source. One may try to use a longer wavelength (above $1100 \mathrm{~nm}$ ) to suppress SPA in the substrate, but since femtosecond pulses are required to induce 3PA in the $\mathrm{GaN}$, those pulses would also generate 2PA in the substrate, which would not help in separating the contributions. One may also try to use longer pulse durations, to eliminate the 3PA effect, for example using picosecond pulses as commonly used for Si testing. However, in this case, no charge would be directly generated in the active layer.

\section{4) Influence of $V_{G S}$ and $V_{D S}$}

Fig. 14 shows that the collected charge associated with long transients, measured at the gate contact, starts to increase with $V_{D S}$ above $500 \mathrm{~V}$. The points for $V_{D S}$ below $500 \mathrm{~V}$ correspond to the noise floor as no long transient was observed at those voltages for a laser pulse energy of $4.4 \mathrm{~nJ}$. This voltage threshold for observing long transients probably reflects the existence of a threshold in the drain-driven electrical field for the activation of the charge transfer mechanism involved in long transients.

The influence of $V_{G S}$ is presented in Fig. 15. As it was observed for the short transient amplitudes, the over-blocking condition reduces the collected charge at the gate, with a plateau from -2 to $0.5 \mathrm{~V}$ and maximum for $\mathrm{V}_{\mathrm{GS}}=0 \mathrm{~V}$.

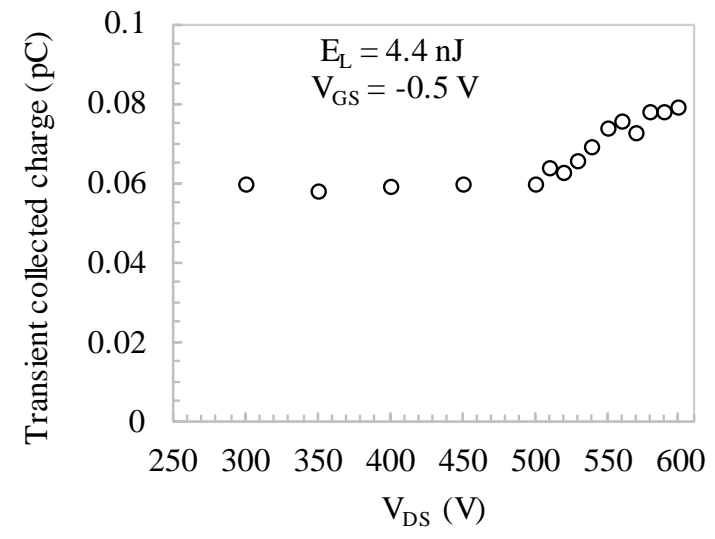

Fig. 14. Long transient collected charge as a function of $V_{D S}$, ref $B$.

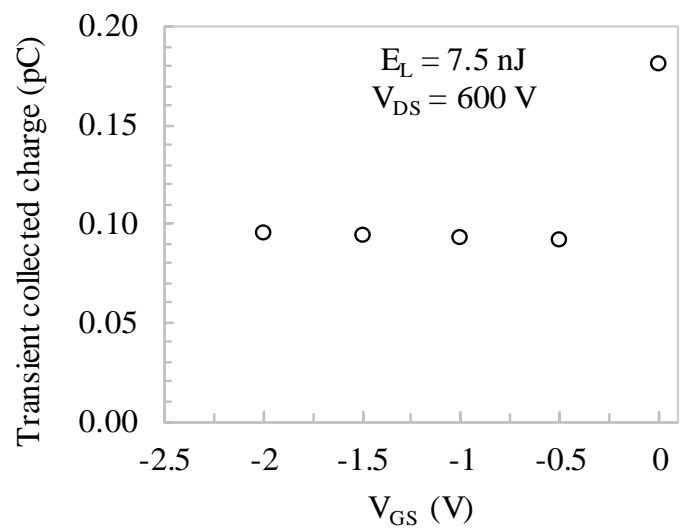

Fig. 15. Long transient collected charge as a function of $\mathrm{V}_{\mathrm{GS}}$, ref $\mathrm{B}$.

\section{Destructive events}

Static drain and gate currents were monitored during the experiments to observe any progressive or sudden degradation of the device. Only sudden destructive events were observed on both references at high $V_{D S}$ when increasing the laser pulse 
energy.

Fig. 16 presents an interrupted mapping with a typical large destructive defect in the background image, generated when hitting the field-plate region that gave the maximum signal in Fig. 11. Such visible destruction was systematically associated with an increase of the static gate and drain leakage currents by at least two orders of magnitude.

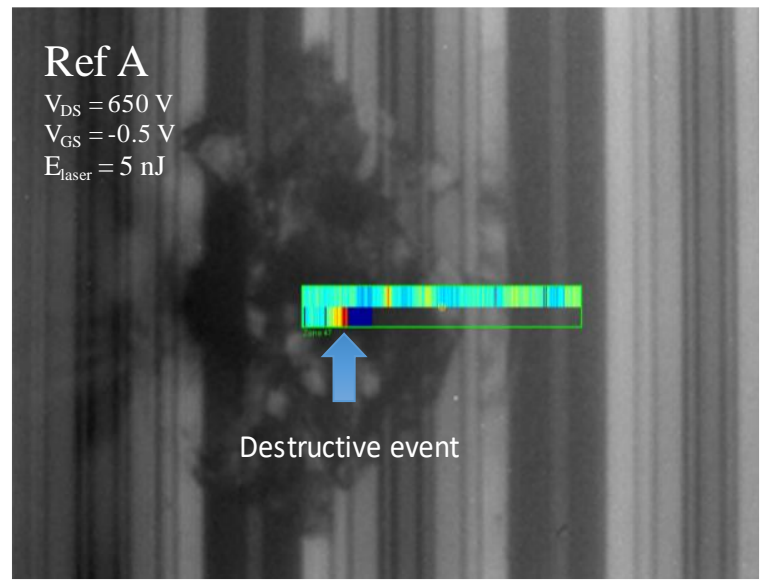

Fig. 16. Visible destructive event triggered when hitting the field plate 2 on ref $\mathrm{A}$.

On other samples, destructive events were observed as a sudden increase of the static gate current by at least one order of magnitude, without any visible degradation of the device in the infrared image.

The electrical signatures of those destructive events are similar to what has been observed under heavy ions in previous works [2]. It is worth noticing that with the laser testing conditions used in this work, no charge was directly generated in the dielectric layers. Note that a detailed root cause analysis of destructive events such as the one in Fig. 16 and its comparison with the failure analysis presented in [2] is made difficult by the large damaged areas. Still, those results demonstrate the possibility to trigger destructive events similar to those induced by heavy ions with laser charge injection. This opens the way to using the laser technique for evaluating and comparing the SOA of different GaN-on-Si HEMT technologies, as it has been done for Si power devices. More results on different devices will be needed to evaluate the level of confidence of such method.

\section{CONCLUSION}

We have demonstrated the possibility to perform backside laser testing of $\mathrm{GaN}$ power HEMTS on $\mathrm{Si}$ substrate using optical parameters compatible with simultaneous 3PA generation in the channel and SPA generation in the substrate. The transient responses observed at the gate were compatible with 3PA in the GaN channel at low drain bias and seem to be dominated by SPA in the substrate at higher drain voltage. The technique is promising for evaluating the sensitivity of these recent power technologies to single-event effects. It allows to map and compare the charge collection efficiency of different devices and to generate destructive events as a function of the supply conditions for identifying the SOA.

\section{REFERENCES}

[1] L. Z. Scheick, "Recent Gallium Nitride Power HEMT Single-Event Testing Results," in 2016 IEEE Radiation Effects Data Workshop (REDW), Portland, OR, USA, 2016, pp. 36-41.

[2] M. Zerarka and O. Crepel, "Radiation robustness of normally-off GaN/HEMT power transistors (COTS)," Microelectron. Reliab., vol. 8890, pp. 984-991, Sep. 2018.

[3] F. Roccaforte, P. Fiorenza, G. Greco, R. Lo Nigro, F. Giannazzo, F. Iucolano, and M. Saggio, "Emerging trends in wide band gap semiconductors ( $\mathrm{SiC}$ and $\mathrm{GaN}$ ) technology for power devices," Microelectron. Eng., vol. 187-188, pp. 66-77, Feb. 2018.

[4] G. Sonia, U. Zeimer, L. Wang, M. Weyers, J. Wurfl, G. Trankle, F. Brunner, A. Denker, R. Lossy, M. Mai, J. Opitz-Coutureau, G. Pensl, E. Richter, and J. Schmidt, "Proton and Heavy Ion Irradiation Effects on AlGaN/GaN HFET Devices," IEEE Trans. Nucl. Sci., vol. 53, no. 6, pp. 3661-3666, Dec. 2006.

[5] Z. Lei, H. Guo, M. Tang, C. Zeng, H. Chen, and Z. Zhang, "Heavy ions irradiation effects on $\mathrm{AlGaN} / \mathrm{GaN}$ high electron mobility transistors," in 2016 16th European Conference on Radiation and Its Effects on Components and Systems (RADECS), Bremen, 2016, pp. 46-49.

[6] E. Mizuta, S. Kuboyama, Y. Nakada, A. Takeyama, T. Ohshima, Y. Iwata, and K. Suzuki, "Single-Event Damage Observed in GaN-on-Si HEMTs for Power Control Applications," IEEE Trans. Nucl. Sci., vol. 65, no. 8, pp. 1956-1963, Aug. 2018.

[7] A. Luu, F. Miller, P. Poirot, R. Gaillard, N. Buard, T. Carriere, P. Austin, M. Bafleur, and G. Sarrabayrouse, "SEB characterisation of commercial power MOSFETs with backside laser and heavy ions of different ranges," in 2007 9th European Conference on Radiation and Its Effects on Components and Systems, Deauville, France, 2007, pp. 365.H.2.1 365.H.2.7.

[8] S. Morand, F. Miller, P. Austin, P. Poirot, R. Gaillard, T. Carriere, and N. Buard, "Temperature effects on power MOSFET and IGBT sensitivities toward single events," in 2011 12th European Conference on Radiation and Its Effects on Components and Systems, Seville, Spain, 2011, pp. 109-114.

[9] F. Darracq, N. Mbaye, C. Larue, V. Pouget, S. Azzopardi, E. Lorfevre, F. Bezerra, and D. Lewis, "Imaging the Single Event Burnout sensitive volume of vertical power MOSFETs using the laser Two-Photon Absorption technique," in 2011 12th European Conference on Radiation and Its Effects on Components and Systems, Seville, Spain, 2011, pp. 434-441.

[10] S. P. Buchner, F. Miller, V. Pouget, and D. P. McMorrow, "Pulsed-Laser Testing for Single-Event Effects Investigations," IEEE Trans. Nucl. Sci., vol. 60 , no. 3, pp. 1852-1875, Jun. 2013.

[11] N. Mbaye, V. Pouget, F. Darracq, and D. Lewis, "Characterization and modeling of laser-induced single-event burn-out in $\mathrm{SiC}$ power diodes," Microelectron. Reliab., vol. 53, no. 9-11, pp. 1315-1319, Sep. 2013.

[12] R. A. Johnson, A. F. Witulski, D. R. Ball, K. F. Galloway, A. L. Sternberg, E. Zhang, L. D. Ryder, R. A. Reed, R. D. Schrimpf, J. A. Kozub, J.-M. Lauenstein, and A. Javanainen, "Enhanced Charge Collection in SiC Power MOSFETs Demonstrated by Pulse-Laser TwoPhoton Absorption SEE Experiments," IEEE Trans. Nucl. Sci., vol. 66, no. 7, pp. 1694-1701, Jul. 2019.

[13] A. Khachatrian, N. J. H. Roche, L. B. Ruppalt, J. G. Champlain, S. Buchner, A. D. Koehler, T. J. Anderson, K. D. Hobart, J. H. Warner, and D. Mcmorrow, "Correlation of the Spatial Variation of Single-Event Transient Sensitivity With Thermoreflectance Thermography in $\mathrm{Al}_{\mathrm{x}} \mathrm{Ga}_{1-\mathrm{x}} \mathrm{N} / \mathrm{GaN}$ HEMTs," IEEE Trans. Nucl. Sci., vol. 65, no. 1, pp. 369-375, Jan. 2018.

[14] A. Khachatrian, N. J. H. Roche, S. Buchner, A. D. Koehler, T. J. Anderson, V. Ferlet-Cavrois, M. Muschitiello, D. McMorrow, B. Weaver, and K. D. Hobart, "A Comparison of Single-Event Transients in Pristine and Irradiated $\mathrm{Al}_{0.3} \mathrm{Ga}_{0.7} \mathrm{~N} / \mathrm{GaN}$ HEMTs using Two-Photon Absorption and Heavy Ions," IEEE Trans. Nucl. Sci., vol. 62, no. 6, pp. 2743-2751, Dec. 2015.

[15] N. J. H. Roche, A. Khachatrian, M. King, S. P. Buchner, J. Halles, R. Kaplar, A. Armstrong, I. C. Kizilyalli, P. D. Cunningham, J. S. Melinger, J. H. Warner, and D. McMorrow, "Two-photon absorption pulsed-laser single-event effect technique for GaN materials and the impact of deep level traps on the carrier generation process," in 2016 16th European Conference on Radiation and Its Effects on Components and Systems (RADECS), Bremen, 2016, pp. 457-460.

[16] A. Khachatrian, N. J. H. Roche, S. P. Buchner, A. D. Koehler, T. J. Anderson, K. D. Hobart, D. McMorrow, S. D. LaLumondiere, N. P. 
Wells, J. Bonsall, E. C. Dillingham, P. Karuza, D. L. Brewe, W. T. Lotshaw, S. C. Moss, V. Ferlet-Cavrois, and M. Muschitiello, "Application of a Focused, Pulsed X-Ray Beam to the Investigation of Single-Event Transients in $\mathrm{Al}_{0.3} \mathrm{Ga}_{0.7} \mathrm{~N} / \mathrm{GaN}$ HEMTs," IEEE Trans. Nucl. Sci., vol. 64, no. 1, pp. 97-105, Jan. 2017.

[17] Y.-F. Wu, A. Saxler, M. Moore, R. P. Smith, S. Sheppard, P. M. Chavarkar, T. Wisleder, U. K. Mishra, and P. Parikh, " $30-\mathrm{W} / \mathrm{mm}$ GaN HEMTs by Field Plate Optimization," IEEE Electron Device Lett., vol. 25, no. 3, pp. 117-119, Mar. 2004.

[18] H. Xing, Y. Dora, A. Chini, S. Heikman, S. Keller, and U. K. Mishra, "High Breakdown Voltage AlGaN-GaN HEMTs Achieved by Multiple Field Plates," IEEE Electron Device Lett., vol. 25, no. 4, pp. 161-163, Apr. 2004

[19] E. Bahat-Treidel, O. Hilt, F. Brunner, V. Sidorov, J. Würfl, and G. Tränkle, "AlGaN/GaN/AlGaN DH-HEMTs Breakdown Voltage Enhancement Using Multiple Grating Field Plates (MGFPs)," IEEE Trans. Electron Devices, vol. 57, no. 6, pp. 1208-1216, Jun. 2010.

[20] Y.-W. Lian, Y.-S. Lin, H.-C. Lu, Y.-C. Huang, and S. S. H. Hsu, "AlGaN/GaN HEMTs on Silicon With Hybrid Schottky-Ohmic Drain for High Breakdown Voltage and Low Leakage Current," IEEE Electron Device Lett., vol. 33, no. 7, pp. 973-975, Jul. 2012.

[21] K. Tanaka, T. Morita, H. Umeda, S. Kaneko, M. Kuroda, A. Ikoshi, H. Yamagiwa, H. Okita, M. Hikita, M. Yanagihara, Y. Uemoto, S. Takahashi, H. Ueno, H. Ishida, M. Ishida, and T. Ueda, "Suppression of current collapse by hole injection from drain in a normally-off GaNbased hybrid-drain-embedded gate injection transistor," Appl. Phys. Lett., vol. 107, no. 16, pp. 163502 1-163502 4, Oct. 2015.

[22] D. Lewis, V. Pouget, F. Beaudoin, P. Perdu, H. Lapuyade, P. Fouillat, and A. Touboul, "Backside laser testing of ICs for SET sensitivity evaluation," IEEE Trans. Nucl. Sci., vol. 48, no. 6, pp. 2193-2201, Dec. 2001.

[23] G. de Graaf and R. F. Wolffenbuttel, "Illumination Source Identification Using a CMOS Optical Microsystem," IEEE Trans. Instrum. Meas., vol. 53, no. 2, pp. 238-242, Apr. 2004

[24] G. E. Jellison, J. D. Budai, C. J. J. C. Bennett, J. Z. Z. Tischler, C. E. Duty, V. Yelundur, and A. Rohatgi, "High-resolution x-ray and light beam induced current (LBIC) measurements of multcrystalline silicon solar cells," in 2010 35th IEEE Photovoltaic Specialists Conference, Honolulu, HI, USA, 2010, pp. 001715-001720.

[25] B. S. Wherrett, "Scaling rules for multiphoton interband absorption in semiconductors," J. Opt. Soc. Am. B, vol. 1, no. 1, pp. 67-72, Mar. 1984.

[26] V. Pouget, "Facilities and methods for radiation testing," in 2014 IEEE NSREC Short Course Notebook, 2014, p. IV II 1-IV II 43.

[27] H. A. MacLeod, Thin-Film Optical Filters, 4th ed. Boca Raton, FL, USA, 2010.

[28] K. Shao, A. Morisset, V. Pouget, E. Faraud, C. Larue, D. Lewis, and D. McMorrow, "3D knife-edge characterization of two-photon absorption volume in silicon for integrated circuit testing," Opt. Express, vol. 19, no. 23, pp. 22594-22599, Nov. 2011.

[29] A. Khachatrian, S. Buchner, A. Koehler, C. Affouda, D. McMorrow, S. D. LaLumondiere, E. C. Dillingham, J. P. Bonsall, A. C. Scofield, and D. L. Brewe, "The Effect of the Gate-Connected Field Plate on SingleEvent Transients in AlGaN/GaN Schottky-Gate HEMTs," IEEE Trans. Nucl. Sci., vol. 66, no. 7, pp. 1682-1687, Jul. 2019. 